\title{
Characterization of a Free-Molecular Pressure Probe employing Gas-Kinetic Simulation
}

\author{
Martin Grabe, Georg Dettleff, Rolf-D. Boettcher, Klaus Hannemann
}

\begin{abstract}
Free-molecular pressure probes are employed to deduce particle flux in highly rarefied flow fields, such as the farfield or the backflow region of a plume expanding into vacuum. Analytical approaches to model the probe behavior are known in the literature for some time now. Numerical methods such as DSMC however allow to conduct investigations into the probe response in regimes inaccessible to analytical approaches. Besides, the numerical results may be compared to theoretical models in order to test their fidelity. We computed the response of a probe with slot orifice to a highly rarefied, parallel flow with varying molecular speed ratio. We confirmed the negligible influence of the speed ratio on probe response for low angles of attack, but observed significant deviations from theory as the angle of attack approaches $90^{\circ}$.
\end{abstract}

\section{Introduction}

The unique DLR High-Vacuum Plume Test Facility (STG) [1] in the Spacecraft Section of the DLR Institute of Aerodynamics and Flow Technology allows to investigate plume expansion from real chemical thrusters in a space-like environment, i. e. the high vacuum conditions can be maintained during thruster operation.

Plume structure and complex configurations are typically investigated employing inert gases in scaled geometries to reduce the number of parameters and in order to increase available measurement time. A free-molecular pressure probe (also referred to as "Patterson-probe" in the literature) is employed to deduce particle flux in the highly rarefied regions of the plume.

Evaluation of the raw probe data requires an involved theory that is strictly applicable only in a high Knudsen number limit, where free-stream particle mean free paths are very large compared to probe dimensions, and intermolecular collisions

Grabe, Dettleff, Boettcher, Hannemann

DLR, Bunsenstr. 10, 37073 Göttingen, Germany, e-mail: martin.grabe@dlr.de 
are negligible. The earliest analytical works assumed idealized probes with infinitely thin walls and infinitely small orifices [2]. The impact of finite wall thickness was subsequently quantified for low speed flows $[3,4]$ using Clausing's approach for free-molecular flow through circular tubes of arbitrary length [5]. A theory for the free-molecular impact probe with circular orifice at arbitrary angles of attack and all speed ratios ("molecular Mach number") was later given by Hughes [6].

Koppenwallner [7] modified the probe design to feature a slit instead of an orifice, and gives engineering approximations to the probe function in the high Mach number / high Knudsen number limit. A much more detailed approach to determine the probe function in free-molecular flow is given by Plähn [8], the evaluation of which already requires numerical methods.

Recent experiments in STG investigate plume-plume interactions, where freemolecular flow conditions cannot be safely inferred from the reservoir conditions and distance to the plume sources. It is today feasible, however, to conduct numerical studies with gas-kinetic simulation methods in flow regimes inaccessible to analytical approaches.

The purpose of this paper is thus to study the deviations from theoretical predictions that must be anticipated when employing a slit-orifice impact probe with finite wall thickness to investigate high Mach number flows with a high degree of rarefaction.

\section{Theory and Methods}

Even though the physical problem is conceivably simple, its analysis is quite involved. We intent to reproduce here only those equations that are absolutely necessary to obtain the results given in the following section. For a more detailed treatment we refer to the original works of Patterson's group, especially $[2,6]$.

\subsection{Description of the free-molecular pressure probe}

The free-molecular pressure probe traditionally consists of a hollow cylinder, closed at one end. The other end is attached to a pressure gauge suitable for high vacuum. To minimize the effect of walls on the entering flow, the probe orifice is located on the longitudinal side of the cylinder (conveniently placed near the closed end). The length of the channel between ambient and internal flow is thus reduced to approximately the wall thickness of the cylinder. Consequently, the probe cylinder is placed perpendicular to the flow.

The measured quantities are: pressure $p_{\mathrm{g}}$ and temperature $T_{\mathrm{g}}$ at the gauge, and temperature $T_{\mathrm{p}}$ at the tip (closed end) of the probe. From these, one infers the pressure $p_{\mathrm{p}}$ at the tip of the probe from a relation 


$$
\frac{p_{\mathrm{p}}}{p_{\mathrm{g}}}=\left(\frac{T_{\mathrm{p}}}{T_{\mathrm{g}}}\right)^{\frac{1}{2}+\omega}
$$

where $\omega \in\left[0, \frac{1}{2}\right]$, depending on the degree of rarefaction inside the cylinder.

A particularly interesting feature of the probe described here is that it may be conveniently rotated around its axis perpendicular to the flow. One may thus obtain several measurements at various angles at a single location of the flow field.

\subsection{Simplifications}

We assume the probe to be exposed to a uniform, parallel free-stream flow with particle density $n_{\infty}$, velocity $u_{\infty}$ and static temperature $T_{\infty}$. Following previous investigations, we model the probe's tip as essentially two-dimensional (Fig. 1). We

Fig. 1 Plane of investigation is perpendicular to the probe major axis (left). The flow may be characterized by its free-stream particle density $n_{\infty}$, bulk velocity $u_{\infty}$ and static temperature $T_{\infty}$. The angle of attack is named $\alpha, p_{\mathrm{p}}$ is the pressure inside the probe.

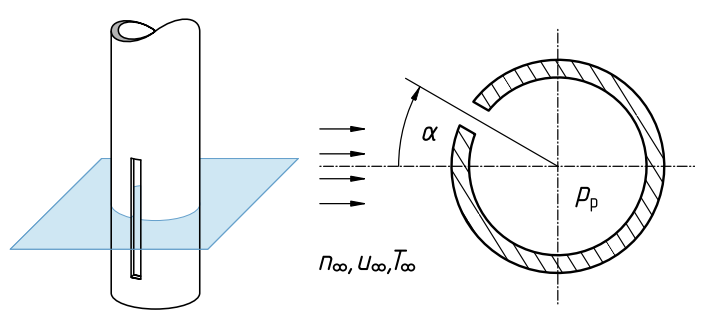

generally investigate only stead-state behavior of the probe, neglecting effects such as outgassing.

\subsection{The ideal probe in free molecular flow}

The concept of the free-molecular pressure probe is best explained in its ideal representation, i. e. neglecting wall thickness and finite orifice width. In the absence of intermolecular collisions, we can clearly distinguish two non-interfering fluxes at the orifice, one of the entering molecules and one of the particles effusing from within the probe. It is an obvious requirement that the two fluxes must be equal in steady state. If we denote by $\dot{n}_{\infty, \mathrm{p}}$ the number of particles per unit time per unit area that enter the probe:

$$
\begin{aligned}
\dot{n}_{\infty, \mathrm{p}} \stackrel{!}{=} \dot{n}_{\mathrm{p}, \infty} & \\
\chi(S, \alpha) \cdot n_{\infty} \tilde{c}_{\infty} & =n_{\mathrm{p}} \tilde{c}_{\mathrm{p}}
\end{aligned}
$$

where 


$$
\chi\left(S_{\infty}, \alpha\right)=\exp \left(-S_{\infty}^{2} \cos ^{2} \alpha\right)+\sqrt{\pi} S_{\infty} \cos \alpha\left(1+\operatorname{erf}\left(S_{\infty} \cos \alpha\right)\right)
$$

is the well-known equilibrium flux function, dependent only on the angle of attack $\alpha$ and the molecular speed ratio $S_{\infty}$ :

$$
S_{\infty}=\frac{u_{\infty}}{\tilde{c}_{\infty}}
$$

The quantity $\tilde{c}$ in Eqs. $(3,5)$ designates the most probable thermal speed in equilibrium, it is directly proportional to the square-root of temperature and otherwise only depends on the gas species. That said, one frequently [6,9] finds Eq. (3) expressed as:

$$
\left(\frac{n_{\mathrm{p}}}{n_{\infty}}\right)_{\text {id }} \sqrt{\frac{T_{\mathrm{p}}}{T_{\infty}}}=\left(\frac{p_{\mathrm{p}}}{p_{\infty}}\right)_{\text {id }} \sqrt{\frac{T_{\infty}}{T_{\mathrm{p}}}}=\chi\left(S_{\infty}, \alpha\right),
$$

where the subscript "id" refers to the "ideal" probe.

\subsection{Effects of finite dimensions}

For real probes with finite wall thickness $L$ and orifice width $W$ the above simplified relations can at best serve as approximations. The channel aspect ratio $\Lambda=W / L$ is now an important parameter. Introducing transmission probability $P(\Lambda)$ as the fraction of particles originally entering a channel and reaching its other end, Eq. (2) reads:

$$
\dot{n}_{\infty}=\frac{P_{\mathrm{p}, \infty}}{P_{\infty, \mathrm{p}}} \cdot \dot{n}_{\mathrm{p}} .
$$

Note, however, that the concept of a transmission probability, as a function of $\Lambda$ only, still presumes free-molecular flow in the channel geometry. We will designate the probability ratio of Eq. (7) by the letter $\Phi$.

Following the above arguments, it is easy to see that $\Phi$ may be conveniently expressed as

$$
\Phi(S, \alpha, \Lambda)=\frac{\left(n_{\mathrm{p}}\right)_{\mathrm{id}}}{n_{\mathrm{p}}}=\frac{\left(p_{\mathrm{p}}\right)_{\mathrm{id}}}{p_{\mathrm{p}}},
$$

which lends itself well to numerical computation. Note that formally one may calculate $\Phi$ also for less rarefied flows, an additional dependence on the degree of rarefaction must then be expected.

\subsubsection{Analytical estimates}

Koppenwallner [7] derived easy-to-use approximations for the transmission probabilities $P_{\mathrm{p}, \infty}, P_{\infty, \mathrm{p}}$ of short slot orifices, applicable to free-molecule flow. For an aspect ratio $\Lambda>1$, these approximations are: 


$$
P_{\mathrm{p}, \infty}=\left.P\right|_{S=0}=\frac{2 \Lambda}{1+2 \Lambda},
$$

and, in the hypersonic limit,

$$
P_{\infty, \mathrm{p}}=\left.P\right|_{S \rightarrow \infty}=\left\{\begin{array}{cl}
1-\frac{\tan \alpha}{2 \Lambda} & \text { if } \quad 0<|\alpha|<\alpha_{\mathrm{lim}}, \\
1 / 2 & \text { else. }
\end{array}\right.
$$

Fig. 2 Geometric properties of the probe orifice: channel length $L$, width $W$ and the resulting limit angle $\alpha_{\text {lim. }}$.

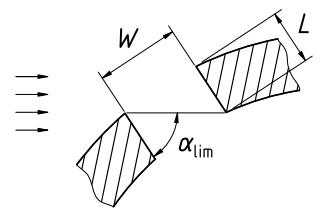

The limit angle $\alpha_{\text {lim }}$ in Eq. (10) results from purely geometrical considerations: it is the angle-of-attack at which no molecule can enter the probe directly, i. e. without prior collision with the channel wall (Fig. 2). Obviously, $\alpha_{\lim }=\arctan (1 / \Lambda)$.

\subsection{Numerical Characterization}

In contrast to previous work $[8,9,6]$, we do not numerically evaluate integral expressions arising from geometrical considerations in free-molecular flow. Instead, we employ the well-known Direct Simulation Monte-Carlo (DSMC) method to model the particle flow and interaction with the probe. The DSMC method is exhaustively described in Bird's monograph [10], but it is already descriptive to imagine DSMC as a virtual experiment, in which a large number of particles move through a domain and collide with each other and with walls, exchanging momentum and energy. Collision processes are modelled stochastically, and steady-state flow field solutions are obtained by sampling averages of particle properties over a large number of decorrelated time intervals. We use the LasVegas implementation of Laux [11].

The degree of rarefaction is commonly quantified by the Knudsen number Kn, which relates the molecular mean free path to a characteristic length of the problem. Unless otherwise stated, we use the mean free path of the undisturbed free stream and non-dimensionalize it with the probe's slit width.

$$
\mathrm{Kn}_{\infty}=\frac{\lambda_{\infty}}{W} .
$$

The simulations presented here were conducted for a $2 \mathrm{D}$ flow in the measurement plane of the cylindrical "DLR Type B" probe, which features a channel aspect ratio of $\Lambda=8 / 5$. The ratio of outer diameter to $L$ is 12 . We vary the angle of attack $\alpha=0^{\circ} \ldots 90^{\circ}$ in steps of $\Delta \alpha=10^{\circ}$. 
The gas is modelled with a Variable Hard Sphere (VHS) intermolecular potential with the parameters of Argon suggested in [10]. All walls are isothermal at stagnation temperature, particles scatter diffusely with full thermal accommodation.

\section{Results}

The probe response to parallel flow characterized by free-stream molecular speed ratios $S_{\infty}=2,5,10$ was investigated. The ratio of transmission probabilities $\Phi$ is evaluated according to Eq. (8). Please recall the practical significance of $\Phi$ as the ratio of actual free-stream particle flux (to be calculated) related to the efflux rate that may be computed from the probe's pressure and temperature readings, cf. Eq. (7).

Fig. 3 Ratio of transmission probabilities $\Phi$ versus angle of attack $\alpha$ for highly rarefied flow $\left(\mathrm{Kn}_{\infty}=1000\right)$ at molecular speed ratios $S_{\infty}=2,5,20$. Symbols represent DSMC calculations, solid lines are interpolations to aid readability.

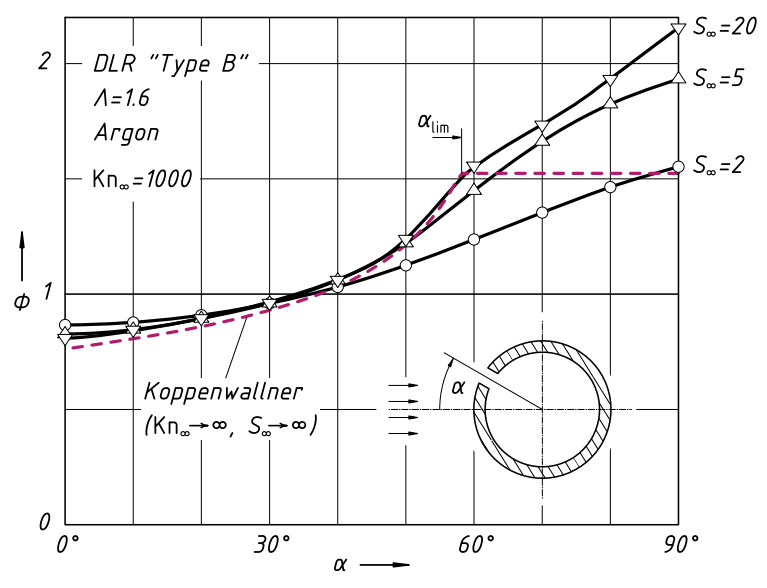

Figure 3 shows the variation of the probability ratio $\Phi$ with the angle of attack $\alpha$ for a degree of rarefaction in which intermolecular collisions are highly unlikely. The computed results shown are parametrized by the molecular speed ratio $S_{\infty}$ of the undisturbed free stream. The dashed line shows the ratio of transmission probabilities computed from Koppenwallner's approximations, Eqs. (9, 10). The agreement with the high speed flow solutions $\left(S_{\infty}=20\right)$ is very good up to the geometrical limiting angle $\alpha_{\mathrm{lim}}$, but deviates substantially after.

Observe that the influence of speed ratio is negligible up to about $\alpha=40^{\circ}$ for the speed range investigated, and remains of low importance in high speed flow $S_{\infty}>5$ even for greater angles. 


\section{Discussion}

The free-molecular nature of the flow in which the results of Fig. 3 are obtained, allows a discussion on purely geometrical arguments. It is helpful for this purpose to introduce the "molecular Mach angle", which is the half-angle of the conical domain in which the actual path of an individual particle is most likely to be found due to the thermal motion superimposed on the bulk velocity. One defines for $S>0$ :

$$
\mu(S)=\arctan \frac{1}{S}
$$

For the speed ratios discussed here we have:

$$
\mu(2) \approx 26.6^{\mathrm{o}}, \quad \mu(5) \approx 11.3^{\mathrm{o}}, \quad \mu(20) \approx 2.9^{\mathrm{o}}
$$

The probability for a highly rarefied, effusing gas to pass through a channel only depends on the channel geometry. As the latter is kept constant in this investigation, the variation of $\Phi$ in Fig. 3 may be discussed in terms of the probability $P_{\infty, \mathrm{p}}$ of particles entering the orifice and eventually reaching the probe cavity. As $P_{\infty, \mathrm{p}}$ is inversely proportional to $\Phi$, incoming molecules are more likely to pass the orifice than effusing ones for probe angles up to $\alpha \approx 35^{\circ}$. This seems comprehensible, as the incoming particles are strongly directed and at low angles of attack may enter the probe without significant wall contact.

As the orifice area perpendicular to the flow decreases with increasing angle of attack, the ratio of particles that can enter the probe directly decreases and the contribution of indirect scattering becomes notable. By indirect scattering we mean the contribution of particles hitting the side walls of the orifice channel at least once and eventually reach the probe cavity, because the direction of their diffuse reemission points towards it. The share of indirectly scattered particles can be expected to depend on the "molecular Mach angle" and this effect is clearly exhibited in Fig. 3 by the divergence of the curves.

At and above the limiting angle of attack $\alpha_{\text {lim }}$ particles can enter the probe directly only by virtue of their thermal component and thus are more likely to do so for low mach numbers. For $\alpha>\alpha_{\lim }+\mu(S)$ indirect collisions dominate and this becomes notable in the curve progression.

Perhaps the most significant result is the obvious deviation of the data for $S_{\infty}=20$ from the theoretical prediction for $\alpha>\alpha_{\text {lim }}$ at $S_{\infty} \rightarrow \infty$. The result obtained from the DSMC calculation seems more plausible however, as the effective wall area available for first impact continues to decrease with increasing angle of attack, thus effectively reducing the transmission probability for entering molecules. 


\section{Conclusions}

From the discussion above we may conclude that the DSMC computations gave a result consistent with expectations and analytical methods at least for angles of attack $\alpha \leq \alpha_{\text {lim }}$. The discrepancy apparent in Fig. 3 between numerical solution and theoretical prediction for high Mach number flow suggests that perhaps the theory is overly simplified for $\alpha>\alpha_{\text {lim. }}$. It is frequently argued [9, 7] that macroscopic properties of the free stream, such as the speed ratio, are readily computed from the ratio of probe pressures $p_{\mathrm{p}}\left(0^{\circ}\right) / p_{\mathrm{p}}\left(90^{\circ}\right)$. Given the large deviations of theoretical from numerical results for the ratio of transmission probabilities at $\alpha=90^{\circ}$, we deem it worthwhile to look more closely into the reasons for the apparent difference in future work.

Figure 3 also suggests that the ratio of transmission probabilities is largely independent of the molecular speed ratio (at least for $S_{\infty} \geq 2$ ) as long as $\alpha$ is less than $\approx 40^{\circ}$. It is thus safe to apply the theoretical methods when investigating rarefied flows at low supersonic speeds, such as backflow occurring in plume-plume interaction problems.

Acknowledgements The authors like to thank Ms. Burnett at the University of Toronto Institute for Aerospace Studies for kindly providing copies of the relevant UTIAS reports.

\section{References}

1. G. Dettleff, K. Plähn, in 33rd Joint Propulsion Conference and Exhibit (Seattle, 1997), AIAA 97-3297

2. G. Patterson, Theory of free-molecule orifice-type pressure probes in isentropic and nonisentropic flows. UTIAS Report 41, University of Toronto Institute for Aerospace Studies, Toronto (1956)

3. E. Harris, G. Patterson, Properties of impact pressure probes in free molecule flow. UTIAS Report 52, University of Toronto Institute for Aerospace Studies, Toronto (1958)

4. K. Enkenhus, E. Harris, G. Patterson, Pressure probes in free molecular flow. UTIAS Report 62, University of Toronto Institute for Aerospace Studies, Toronto (1959)

5. P. Clausing, Annalen der Physik 404(8), 961 (1932). DOI 10.1002/andp.19324040804

6. P. Hughes, Theory for the free molecular impact probe at angle of attack. UTIAS Report 103, University of Toronto Institute for Aerospace Studies, Toronto (1965)

7. G. Koppenwallner, in Rarefied Gas Dynamics, ed. by H. Oguchi (University of Tokyo Press, Tsukuba, 1984), no. 14 in International Symposium on Rarefied Gas Dynamics, pp. 415-422

8. K. Plähn, Experimentelle Untersuchung und Modellierung von Abgasstrahlen aus Kleintriebwerken in der Kryo-Vakuum-Anlage STG. Ph.D. thesis, Universität Hannover (1999). DLR Forschungsbericht 1999-39

9. K. Bier, W. Ehrfeld, Zur Untersuchung von Strömungen verdünnter Gase mit MiniaturDrucksonden. KFK-Report 842, Institut für Kernverfahrenstechnik, Karlsruhe (1968)

10. G.A. Bird, Molecular Gas Dynamics and the Direct Simulation of Gas Flows (Oxford University Press, 1994)

11. M. Laux, Direkte Simulation verdünnter, reagierender Strömungen. Ph.D. thesis, Institut für Raumfahrtsysteme, Universität Stuttgart (1996) 